\title{
Regenerative effect of recombinant human bone morphogenetic protein-2/absorbable collagen sponge (rhBMP-2/ACS) after sequestrectomy of medication-related osteonecrosis of the jaw (MRONJ)
}

\author{
Song-Hee Min, No-Eul Kang, Seung-II Song, Jeong-Keun Lee \\ Department of Oral and Maxillofacial Surgery, Institute of Oral Health Science, Ajou University School of Medicine, Suwon, Korea
}

\begin{abstract}
J Korean Assoc Oral Maxillofac Surg 2020;46:191-196)
Objectives: Beyond the original application approved by the U.S. Food and Drug Administration, recombinant human bone morphogenetic protein-2 (rhBMP-2) is used for medication-related osteonecrosis of the jaw (MRONJ) treatment because of its bone remodeling enhancement properties. The purpose of the study was to investigate the bone formation effect of rhBMP-2/absorbable collagen sponge (ACS) in patients with MRONJ. Materials and Methods: In this retrospective cohort study, 26 female patients diagnosed with MRONJ and who underwent mandibular sequestrectomy at Ajou University Dental Hospital from 2010 to 2018 were included. The experimental group was composed of 18 patients who received rhBMP-2/ACS after sequestrectomy, while the control group was composed of 8 patients who did not receive rhBMP-2/ACS after sequestrectomy. A total dose of $0.5 \mathrm{mg}$ of rhBMP-2 was used in the experimental group at a concentration of $0.5 \mathrm{mg} / \mathrm{mL}$. Follow-up panoramic X-rays were taken immediately after the surgery and more than 6 months after the surgery. Using those X-rays, a radiographic index of bone defect area was calculated using the modified Ihan Hren method, which measures radiographic density of the normal bone and the defect site. Results: This study suggests that rhBMP-2 contributes to new bone formation. The mean radiographic index immediately after surgery and more than 6 months after the surgery for the experimental group was $68.4 \%$ and $79.8 \%$, respectively. The mean radiographic index immediately after surgery and more than 6 months after the surgery for the control group was $73.4 \%$ and $76.7 \%$, respectively (Wilcoxon signed rank test, $P>0.05$ ). The mean radiographic index increased $11.4 \%$ in the experimental group and $3.27 \%$ in the control group (Mann-Whitney U-test, $P<0.05$ ). Conclusion: Based on the results, use of rhBMP-2/ACS on bone defect sites after sequestrectomy could be a successful strategy for treatment of MRONJ patients.
\end{abstract}

Key words: Recombinant human bone morphogenetic protein-2, Osteomyelitis, Bisphosphonate-associated osteonecrosis of the jaw, Osteonecrosis [paper submitted 2019. 10. 16 / revised 2020. 2. 10 / accepted 2020. 2. 25]

\section{Introduction}

Osteonecrosis of the jaw (ONJ) was a rare disease until the advent of anti-osteoporotic drugs, use of which results in medication-related osteonecrosis of the jaw (MRONJ). This is the most common form of ONJ, exhibiting a $0.04 \%$ incidence in Korea ${ }^{1}$. Moreover, the incidence is much higher

\footnotetext{
Jeong-Keun Lee

Department of Oral and Maxillofacial Surgery, Institute of Oral Health Science, Ajou University School of Medicine, 164 WorldCup-ro, Yeongtonggu, Suwon 16499, Korea

TEL: +82-31-219-5328 FAX: +82-31-219-5329

E-mail:arcady@ajou.ac.kr

ORCID: https://orcid.org/0000-0002-5561-6297

(a) This is an open-access article distributed under the terms of the Creative Commons Attribution Non-Commercial License (http://creativecommons.org/ licenses/by-nc/4.0/), which permits unrestricted non-commercial use, distribution, and reproduction in any medium, provided the original work is properly cited. Copyright (C) 2020 The Korean Association of Oral and Maxillofacial Surgeons. All rights reserved.
}

in patients undergoing surgical dental treatments, making it a significant concern for most dentists.

The diagnostic criteria for MRONJ are as follows: (1) current or previous treatment with antiresorptive or antiangiogenic agent, (2) exposed bone or bone that can be probed through an intraoral or extra-oral fistula in the maxillofacial region that has persisted for more than eight weeks, and (3) no history of radiation therapy to the jaw or obvious metastatic disease to the jaw ${ }^{2}$.

There is no standard approach for treating MRONJ in the literature. Some authors have recommended a conservative approach to MRONJ ${ }^{3,4}$. Gómez Font et al. ${ }^{3}$ recommended long-term antibiotic treatment and chlorhexidine rinses 3 to 4 times a day in the MRONJ update, excluding aggressive surgical treatment. Ruggiero et al. ${ }^{4}$ suggested oral antimicrobial rinses combined with antibiotic therapy for stage 1 and 2 patients. In addition, the positional paper of the American 
Association of Oral and Maxillofacial Surgeons (AAOMS) suggests that the surgical procedure be limited to patients with stage 3 disease. However, most MRONJ patients exhibit infection-related symptoms that do not respond well to conservative treatment. There are several studies reporting good results with surgical management of early stages of MRONJ. Vescovi et al. ${ }^{5}$ reported good healing of conservative surgical treatment for stage 1 bisphosphonate-related osteonecrosis of the jaw (BRONJ) patients. The surgical approach could be a good solution in patients not responding to conservative therapy.

Recent studies have focused on use of bone morphogenetic protein-2 (BMP-2) for MRONJ treatment ${ }^{6-8}$. Originally, the U.S. Food and Drug Administration (FDA) approved recombinant human BMP-2 (rhBMP-2) as a synthetic bone graft material for maxillary sinus augmentation and socket preservation based on its osteoinductive properties ${ }^{9}$. Several studies have since demonstrated that rhBMP-2 works in situations beyond the aforementioned applications ${ }^{10-12}$.

For optimal effect, rhBMP-2 should be bound to the appropriate scaffolds to stabilize its release into the lesion. These scaffolds include hydroxyapatite, tricalcium phosphate, demineralized bone matrix, and absorbable collagen sponge (ACS). Li and Wozney ${ }^{13}$ reported that the release period of rhBMP-2 was more than twice as long when ACS was applied compared to when there was no scaffold, supporting ACS as a suitable scaffold for rhBMP-2 applications ${ }^{13,14}$.

The major pharmacological effect of bisphosphonates is postulated to be inhibition of osteoclastic activity. Considering this with the hypothesis that the pathogenesis of MRONJ involves oversuppression of bone remodeling ${ }^{15}$, BMPs are expected to promote the healing of bone in MRONJ patients by enhancing bone remodeling.

The purpose of this study was to investigate the regenerative effect of rhBMP-2/ACS in patients with MRONJ. The authors theorize that rhBMP-2 will promote bone healing in
MRONJ, and the study involved a quantitative evaluation of the regenerative effect of rhBMP-2/ACS after sequestrectomy in MRONJ patients.

\section{Materials and Methods}

\section{Patients}

Twenty-six patients were included in this investigation. All the patients were diagnosed with MRONJ, underwent sequestrectomy at Ajou University Dental Hospital from 2010 to 2018, and were followed for more than 6 months. This study was conducted after approval from the Institutional Review Board of Ajou University Hospital (IRB No. AJIRB-MEDMDB-19-375), and the informed consent was waived. For reliability of data analysis, female patients and patients who underwent mandible site surgery were included. Based on the MRONJ diagnostic principle announced by the AAOMS, a patient was diagnosed with MRONJ when bone was exposed or probed through the fistula for more than eight weeks with no history of radiation therapy to the jaw or a history of definite metastatic disease.

All the patients were divided into two groups. Patients provided with application of rhBMP-2/ACS in the bone defect site after sequestrectomy were classified as the experimental group, while patients who did not receive rhBMP-2/ACS application in the defect site were classified into the control group.

\section{Surgical procedure}

During surgical treatment, the sequestrum and affected teeth were removed. Curettage was performed until fresh bone was exposed. For the experimental group, ACS (Ateloplug; Bioland, Seoul, Korea) soaked with rhBMP-2 (NOVOSIS; Daewoong Pharmaceutical Company, Seoul, Korea) was
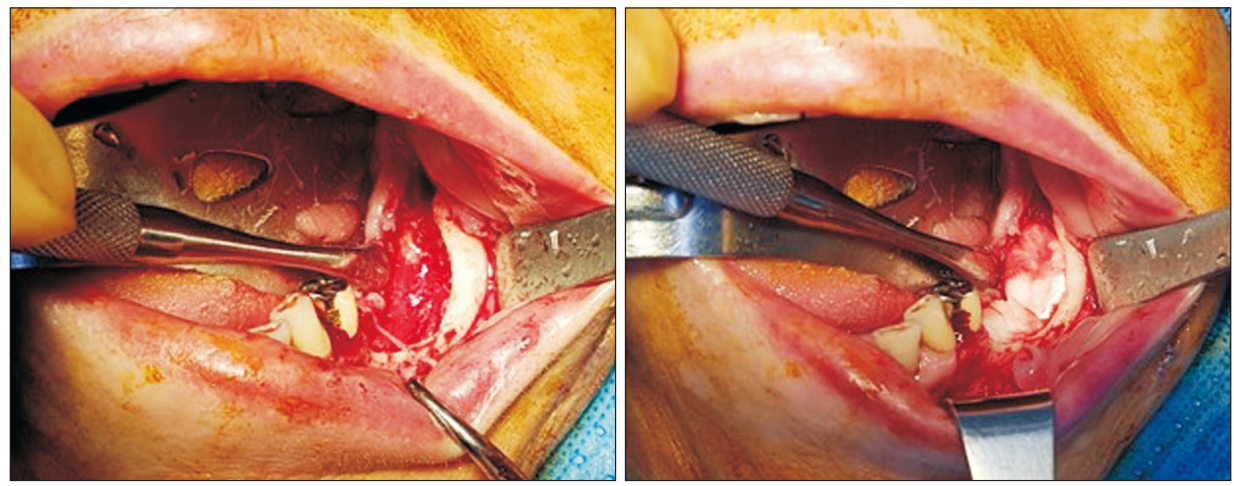

Fig. 1. Surgical procedure of experimental group. Absorbable collagen sponge with absrobed recombinant human bone morphogenetic protein-2 was applied to the bone defect.

Song-Hee Min et al: Regenerative effect of recombinant human bone morphogenetic protein-2/ absorbable collagen sponge (rhBMP-2/ACS) after sequestrectomy of medication-related osteonecrosis of the jaw (MRONJ). J Korean Assoc Oral Maxillofac Surg 2020 
inserted into the defect site, and a primary suture was placed. (Fig. 1) One kit of rhBMP-2 was used for each defect site at a concentration of $0.5 \mathrm{mg} / \mathrm{mL}$, for a total dose of $0.5 \mathrm{mg}$. Various numbers of ACS were used depending on the size of the defect. For the control group, only a primary suture was placed without ACS or rhBMP-2.

\section{Bone density analysis}

In this study, it was assumed that the radiopacity and bone density of the bone defect site in radiographs were proportional. The changes in bone density were estimated using the radiographic density of the panoramic X-ray to analyze the degree of bone healing.
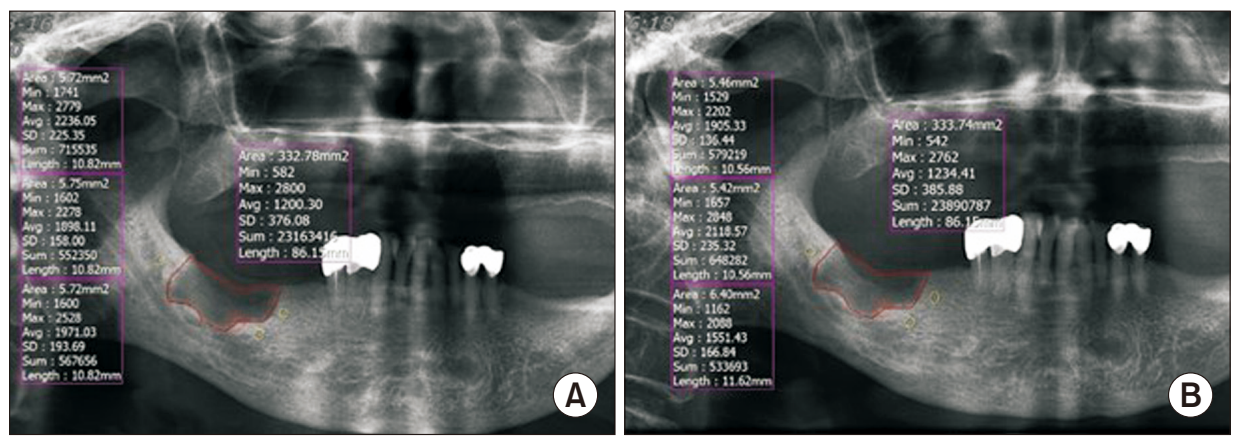

Fig. 2. Bone density analysis method. To measure the radiographic density of the normal bone, the mean value was calculated by measuring each of the three random site around the bone defect area. To measure changes in radiographic density of the bone defect, the bone defect area of the panoramic X-ray was set to the same extent as the panoramic X-ray immediately after surgery, and after six months of surgery. A. Immediately after surgery. B. Postoperation over six months of surgery.

Song-Hee Min et al: Regenerative effect of recombinant human bone morphogenetic protein-2/absorbable collagen sponge (rhBMP-2/ACS) after sequestrectomy of medication-related osteonecrosis of the jaw (MRONJ). J Korean Assoc Oral Maxillofac Surg 2020

Table 1. Patient information of the experimental group

\begin{tabular}{|c|c|c|c|c|c|c|}
\hline Patient No. & Age (yr) & $\operatorname{Sex}$ & $\mathrm{BP}$ & Reason for BP use & $\begin{array}{l}\text { Follow-up } \\
\text { point (mo) }\end{array}$ & $\begin{array}{c}\text { Defect area } \\
\left(\mathrm{mm}^{2}\right)\end{array}$ \\
\hline 1 & 55 & $\mathrm{~F}$ & Zolendronate & Breast cancer (bone meta) & 6 & 250.05 \\
\hline 2 & 77 & $\mathrm{~F}$ & Alendronate & Osteoporosis & 6 & 310.17 \\
\hline 3 & 88 & $\mathrm{~F}$ & Risedronate, ibandronate & Osteoporosis & 6 & 189.49 \\
\hline 4 & 65 & $\mathrm{~F}$ & Alendronate & Osteoporosis & 10 & 179.94 \\
\hline 5 & 91 & $\mathrm{~F}$ & Alendronate, ibandronate & Osteoporosis & 6 & 40.04 \\
\hline 6 & 82 & $\mathrm{~F}$ & Alendronate & Osteoporosis & 6 & 206.62 \\
\hline 7 & 71 & $\mathrm{~F}$ & Risedronate, ibandronate & Osteoporosis & 12 & 76.62 \\
\hline 8 & 76 & $\mathrm{~F}$ & Risedronate & Osteoporosis & 6 & 82.09 \\
\hline 9 & 76 & $\mathrm{~F}$ & Ibandronate & Osteoporosis & 17 & 160.56 \\
\hline 10 & 66 & $\mathrm{~F}$ & Alendronate & Osteoporosis & 6 & 317.31 \\
\hline 11 & 77 & $\mathrm{~F}$ & Ibandronate & Osteoporosis & 12 & 165.44 \\
\hline 12 & 79 & $\mathrm{~F}$ & Unknown BP & Osteoporosis & 6 & 70.58 \\
\hline 13 & 87 & $\mathrm{~F}$ & Zolendronate & Osteoporosis & 6 & 153.34 \\
\hline 14 & 75 & $\mathrm{~F}$ & Risedronate, ibandronate & Osteoporosis & 6 & 257.76 \\
\hline 15 & 73 & $\mathrm{~F}$ & Risedronate & Osteoporosis & 6 & 111.56 \\
\hline 16 & 77 & $\mathrm{~F}$ & Alendronate & Osteoporosis & 7 & 210.62 \\
\hline 17 & 66 & $\mathrm{~F}$ & Alendronate & Osteoporosis & 7 & 157.04 \\
\hline 18 & 67 & $\mathrm{~F}$ & Unknown BP & Osteoporosis & 6 & 353.03 \\
\hline
\end{tabular}

(BP: bisphosphonate, F: female)

Song-Hee Min et al: Regenerative effect of recombinant human bone morphogenetic protein-2/absorbable collagen sponge (rhBMP-2/ACS) after sequestrectomy of medication-related osteonecrosis of the jaw (MRONJ). J Korean Assoc Oral Maxillofac Surg 2020 
Table 2. Patient information of the control group

\begin{tabular}{ccclcrr}
\hline Patient No. & Age $(\mathrm{yr})$ & Sex & \multicolumn{1}{c}{ BP } & Reason for BP use & Follow-up point $(\mathrm{mo})$ & Defect area $\left(\mathrm{mm}^{2}\right)$ \\
\hline 1 & 77 & $\mathrm{~F}$ & Unknown BP & Osteoporosis & 12 & 178.37 \\
2 & 83 & $\mathrm{~F}$ & Alendronate & Osteoporosis & 6 & $1,073.87$ \\
3 & 67 & $\mathrm{~F}$ & Alendronate & Osteoporosis & 6 & 186.16 \\
4 & 76 & $\mathrm{~F}$ & Alendronate & Osteoporosis & 6 & 336.88 \\
5 & 74 & $\mathrm{~F}$ & Alendronate & Osteoporosis & 6 & 79.79 \\
6 & 63 & $\mathrm{~F}$ & Unknown BP & Osteoporosis & 10 & 236.37 \\
7 & 83 & $\mathrm{~F}$ & Alendronate, ibandronate & Osteoporosis & 6 & 145.72 \\
8 & 76 & $\mathrm{~F}$ & Alendronate & & 617.60 \\
\hline
\end{tabular}

(BP: bisphosphonate, F: female)

Song-Hee Min et al: Regenerative effect of recombinant human bone morphogenetic protein-2/absorbable collagen sponge (rhBMP-2/ACS) after sequestrectomy of medication-related osteonecrosis of the jaw (MRONJ). J Korean Assoc Oral Maxillofac Surg 2020

Table 3. Radiographic index

\begin{tabular}{cccc}
\hline Group & $\begin{array}{c}\text { Immediately after } \\
\text { operation }(\%)\end{array}$ & $\begin{array}{c}\text { More than } 6 \text { mo } \\
\text { after operation }(\%)\end{array}$ & $P$-value \\
\hline Experimental & $68.4 \pm 12.9$ & $79.8 \pm 13.0$ & 0.001 \\
Control & $73.4 \pm 13.6$ & $76.7 \pm 12.1$ & 0.401 \\
\hline
\end{tabular}

Values are presented as mean \pm standard deviation.

$P$-value by Wilcoxon signed rank test.

Song-Hee Min et al: Regenerative effect of recombinant human bone morphogenetic protein-2/absorbable collagen sponge (rhBMP-2/ACS) after sequestrectomy of medication-related osteonecrosis of the jaw (MRONJ). J Korean Assoc Oral Maxillofac Surg 2020

Radiographic index $(\%)=$

Radiographic density of the defect site

Radiographic density of the adjacent site $\times 100$

To measure the change in radiographic density, the following process was performed using panoramic X-rays taken after six months. The bone defect area was set as in the panoramic X-ray taken immediately after the surgery.(Fig. 2) The above process was conducted twice by one examiner, and the mean of the measured values was used.

\section{Statistical analysis}

Statistical analysis was performed using IBM SPSS Statistics (ver. 23.0; IBM, Armonk, NY, USA). The Wilcoxon signed rank test was used for analyzing the effectiveness in each group at a significance level of 5\%. The Mann-Whitney U-test was used to analyze the effectiveness between the experimental and control groups at a significance level of 5\%.

\section{Results}

Total number of patients was 26 , with an experimental group of 18 and control group of 8 . The mean age was 74.89 years and 74.88 years for the experimental and control groups, respectively.(Tables 1, 2)
Table 4. Increase of radiographic index

\begin{tabular}{lcc}
\hline \multicolumn{1}{c}{ Group } & Change of radiographic index $(\%)$ & $P$-value \\
\hline Experimental & $11.4 \pm 10.6$ & \multirow{2}{*}{0.026} \\
Control & $3.27 \pm 10.7$ & \\
\hline
\end{tabular}

Values are presented as mean \pm standard deviation.

$P$-value by Mann-Whitney U-test.

Song-Hee Min et al: Regenerative effect of recombinant human bone morphogenetic protein-2/absorbable collagen sponge (rhBMP-2/ACS) after sequestrectomy of medication-related osteonecrosis of the jaw (MRONJ). J Korean Assoc Oral Maxillofac Surg 2020

\section{Radiographic index}

The mean radiographic index for the experimental group was $68.4 \%$ and $79.8 \%$ immediately after surgery and more than 6 months after surgery, respectively. The mean radiographic index for the control group was $73.4 \%$ and $76.7 \%$ immediately after surgery and more than 6 months after surgery, respectively. The Wilcoxon signed rank test was performed to verify the effects of osteogenesis immediately after surgery and 6 months after surgery in each group. As a result, the $P$-value in the experimental group was 0.001 , indicating a statistically significant effect, but a nonsignificant 0.401 in the control group.(Table 3)

\section{Radiographic index increase}

The mean radiographic index increased $11.4 \%$ in the experimental group and $3.27 \%$ in the control group. As a result of the Mann-Whitney U-test, the $P$-value in the experimental group was 0.026 , representing a statistically significant effect. (Table 4)

\section{Discussion}

The purpose of this study was to investigate the bone formation effect of rhBMP-2/ACS in patients with MRONJ. This study theorized that rhBMP-2/ACS could promote bone 
healing by enhancing bone remodeling in MRONJ patients and compared the extent of bone healing with application of rhBMP-2/ACS after sequestrectomy. In this study, the bone density analysis method of bone defect area was based on the radiopacity of panoramic X-rays. This study demonstrated an increase in radiographic index in the experimental group compared to the control group.

The method of bone density analysis in the bone defect area assumes that the radiopacity of the bone around the bone defect will have the same radiopacity as the normal bone. In a study by Ihan Hren and Miljavec ${ }^{16}$, the initial radiographic index of the defect was $88 \%$. In the present study, the mean initial index of the defects was $70.3 \%$ and increased with time. The initial radiographic index difference from that of Ihan Hren and Miljavec's study ${ }^{16}$ is attributed to the differences in the type of defect or the amount of radiation exposure while obtaining panoramic X-rays. Based on the above findings, bone density can be estimated using panoramic X-rays.

Cicciù et al. ${ }^{6}$ investigated the clinical effects of rhBMP-2 without concomitant bone grafting materials in MRONJ patients undergoing sequestrectomy and reported successful healing of the necrotic region. In this study, the experimental group identified an increase in radiographic index 6 months after surgery (Wilcoxon signed rank test, $P<0.05$ ). In addition, there was a statistically significant difference in the radiographic index increase between experimental and control groups (Mann-Whitney U-test, $P<0.05$ ), which may be the result rhBMP-2/ACS facilitating bone healing. This result suggests that rhBMP-2/ACS contributes to new bone formation.

In Ihan Hren and Miljavec's study ${ }^{16}$, the mean increase in radiopacity of the bone defect was $27 \%$ at 6 months after cyst enucleation in healthy patients. In the present study, the mean increase was $11.2 \%$ in the experimental group. Despite using rhBMP-2/ACS, bone regeneration in MRONJ patients is postulated to be difficult compared to that in normal bone.

There are many qualitative studies on the effect of rhBMP-2 in patients with MRONJ but few quantitatively analyzed studies. To our knowledge, the significance of this study is demonstration of the quantitative effect of rhBMP-2 in patients with MRONJ.

This study had the following limitations: small sample sizes, difficulty in determining the margin of bone defects, and panoramic X-ray evaluations providing limited information in bone density evaluations. For more accurate analysis, computed tomography-based studies are recommended.

\section{Conclusion}

When rhBMP-2/ACS was used on bone defect sites after sequestrectomy, the rate of new bone formation increased. Therefore, it use of rhBMP-2/ACS on bone defect sites after sequestrectomy could be a successful strategy in treatment of MRONJ patients. Currently, there is no FDA approval for use of rhBMP-2 for this purpose, but it is expected to have clinical usefulness in the future.

\section{ORCID}

Song-Hee Min, https://orcid.org/0000-0001-8988-2094

No-Eul Kang, https://orcid.org/0000-0001-7870-3257

Seung-Il Song, https://orcid.org/0000-0001-9504-6471

Jeong-Keun Lee, https://orcid.org/0000-0002-5561-6297

\section{Authors' Contributions}

S.H.M. participated in data collection and wrote the manuscript and performed statistical analysis. N.E.K., S.I.S., and J.K.L. participated in the study design and helped to draft the manuscript. All authors read and approved the final manuscript.

\section{Ethics Approval and Consent to Participate}

This study was conducted after approval from the Institutional Review Board of Ajou University Hospital (IRB No. AJIRB-MED-MDB-19-375), and the informed consent was waived.

\section{Conflict of Interest}

No potential conflict of interest relevant to this article was reported.

\section{References}

1. Lee JK, Kim KW, Choi JY, Moon SY, Kim SG, Kim CH, et al. Bisphosphonates-related osteonecrosis of the jaw in Korea: a preliminary report. J Korean Assoc Oral Maxillofac Surg 2013;39:913.

2. Ruggiero SL, Dodson TB, Fantasia J, Goodday R, Aghaloo T, Mehrotra B, et al. American Association of Oral and Maxillofacial Surgeons position paper on medication-related osteonecrosis of the jaw--2014 update. J Oral Maxillofac Surg 2014;72:1938-56.

3. Gómez Font R, Martínez García ML, Olmos Martínez JM. Osteochemonecrosis of the jaws due to bisphosphonate treatments. 
Update. Med Oral Patol Oral Cir Bucal 2008;13:E318-24.

4. Ruggiero SL, Dodson TB, Assael LA, Landesberg R, Marx RE, Mehrotra B. American Association of Oral and Maxillofacial Surgeons. American Association of Oral and Maxillofacial Surgeons position paper on bisphosphonate-related osteonecrosis of the jaw 2009 update. Aust Endod J 2009;35:119-30.

5. Vescovi P, Merigo E, Meleti M, Manfredi M, Fornaini C, Nammour $\mathrm{S}$, et al. Conservative surgical management of stage I bisphosphonate-related osteonecrosis of the jaw. Int J Dent 2014;2014:107690.

6. Cicciù M, Herford AS, Juodžbalys G, Stoffella E. Recombinant human bone morphogenetic protein type 2 application for a possible treatment of bisphosphonates-related osteonecrosis of the jaw. $\mathrm{J}$ Craniofac Surg 2012;23:784-8.

7. Bagan J, Scully C, Sabater V, Jimenez Y. Osteonecrosis of the jaws in patients treated with intravenous bisphosphonates (BRONJ): a concise update. Oral Oncol 2009;45:551-4.

8. Park JH, Kim JW, Kim SJ. Does the addition of bone morphogenetic protein 2 to platelet-rich fibrin improve healing after treatment for medication-related osteonecrosis of the jaw? J Oral Maxillofac Surg 2017;75:1176-84.

9. Freitas RM, Spin-Neto R, Marcantonio Junior E, Pereira LA, Wikesjö UM, Susin C. Alveolar ridge and maxillary sinus augmentation using rhBMP-2: a systematic review. Clin Implant Dent Relat Res 2015;17 Suppl 1:e192-201.

10. Carter TG, Brar PS, Tolas A, Beirne OR. Off-label use of recombinant human bone morphogenetic protein-2 (rhBMP-2) for reconstruction of mandibular bone defects in humans. J Oral Maxillofac Surg 2008;66:1417-25.

11. Hwang DY, On SW, Song SI. Bone regenerative effect of recombinant human bone morphogenetic protein-2 after cyst enucleation.
Version 2. Maxillofac Plast Reconstr Surg 2016;38:22.

12. Chenard KE, Teven CM, He TC, Reid RR. Bone morphogenetic proteins in craniofacial surgery: current techniques, clinical experiences, and the future of personalized stem cell therapy. J Biomed Biotechnol 2012;2012:601549.

13. Li RH, Wozney JM. Delivering on the promise of bone morphogenetic proteins. Trends Biotechnol 2001;19:255-65.

14. Geiger M, Li RH, Friess W. Collagen sponges for bone regeneration with rhBMP-2. Adv Drug Deliv Rev 2003;55:1613-29.

15. Russell RG, Watts NB, Ebetino FH, Rogers MJ. Mechanisms of action of bisphosphonates: similarities and differences and their potential influence on clinical efficacy. Osteoporos Int 2008;19:73359.

16. Ihan Hren N, Miljavec M. Spontaneous bone healing of the large bone defects in the mandible. Int J Oral Maxillofac Surg 2008;37:1111-6.

17. Yim JH, Lee JH. Panoramic analysis about spontaneous bone regeneration after enucleation of jaw cyst. J Korean Assoc Maxillofac Plast Reconstr Surg 2009;31:229-36.

\section{How to cite this article: Min SH, Kang NE, Song SI, Lee JK. Re-} generative effect of recombinant human bone morphogenetic protein-2/absorbable collagen sponge (rhBMP-2/ACS) after sequestrectomy of medication-related osteonecrosis of the jaw (MRONJ).

J Korean Assoc Oral Maxillofac Surg 2020;46:191-196. https://doi. org/10.5125/jkaoms.2020.46.3.191 